\title{
Nasal Aging and Rejuvenation
}

\begin{abstract}
Nasal tissues are formed by bone, cartilage, muscles, subcutaneous layer and skin. Anatomy and physiology have been extensively studied and published; nevertheless little importance has been given to the concept of nasal aging and its related rejuvenation procedures. The author presents a review regarding the principal aspects of nasal aging and an algorithm to approach nasal rejuvenation.
\end{abstract}

Volume 9 Issue 2 - 2017

\author{
Sebastian Torres \\ Plastic and Aesthetic Surgery Private Practice, Chile \\ Correspondence: Sebastian Torres Plastic and Aesthetic \\ Surgery Private Practice Santiago, Chile, \\ Email sebastiantorresmd@gmail.com \\ Received: July 06, 2017 | Published: November 22, 2017
}

\section{Introduction}

Anatomical nasal tissues include bone, cartilage, muscles, subcutaneous layer and skin. All these elements are carefully organized to allow proper physiology and grant adequate aesthetics. These variables have been extensively studied and published, principally related to the correction of development or post traumatic abnormalities. Nasal surgical corrections have focus on reestablishment of normal function and anatomy. Patients seeking nasal surgical corrections are generally young individuals. The former has influenced little interest and development of the concept of nasal aging and its related rejuvenation procedures.

Nevertheless, since 2008, Braccini's concept of medical rhinoplasty has expanded the armamentarium available for the treatment of nasal conditions and the universe of patient seeking corrections. Although the majority of patients seeking medical rhinoplasty are young individuals, there is a new trend in the number of adults and older patients requiring minimally invasive nasal corrections.

The study of nasal aging becomes vital prior to nasal rejuvenation procedures.

\section{Nasal Aging}

Nasal aging includes skin aging and related tissues aging. Skin aging comprises chrono and photo aging. Photo aging is sun UVexposure related and is highly preventable. Changes in the skin due to sun damage include elastosis, melanin disorders (hypo/hyper pigmentation), premalignant and malignant conditions.

Chrono aging is due to the pass of time and may include skin thinning or thickening depending on the case, and sebum and hydration variations.

The rosacea related disorder manifests in the nose as telangiectasia and vascular development, ostium follicularis and skin enlargement that may determine the apparition of rhinophyma. This condition although genetically determined, can be aggravated by the heat or alcohol.

Related tissues of the nose are also prone to aging. Generally with the pass of time, the adipose tissue tends to atrophy, especially in the region of the glabella and root, but also in the dorsum and tip. The former affects directly the exposure of deeper tissues such as cartilage and bone, which get superficialized, giving the nose a skeletonized aspect. This phenomena is particularly important in pre existent thin soft tissues patients, specially females.
The cartilage component experiments a continuous growth of medial and lateral crura, affecting dome projection and rotation. On the contrary caudal septum tends to reabsorb in time. This events although non exclusive are more prominent in males, that present thicker and heavier tissues.

The bony aspect of the nose experiments enlargement of the piriform aperture and reabsorption of the anterior nasal spine, points where the whole structure of the nose is anchored.

All above determines the visual detachment of the nose from the face, as the superior and inferior thirds of the nose get under projected. The nose tip typically becomes larger with a lot of tip defining points, support, projection and rotation. Furthermore, there is a flattening of the supratip break.

Clinically the frontal view of the aging nose has a reduce vision of the nostrils and retracted or hidden columella. Lateral views shows a reduction in Nasolabial angle that are typically under 85 degrees in males and 90 in females. All the former changes are shown in Figure 1 .

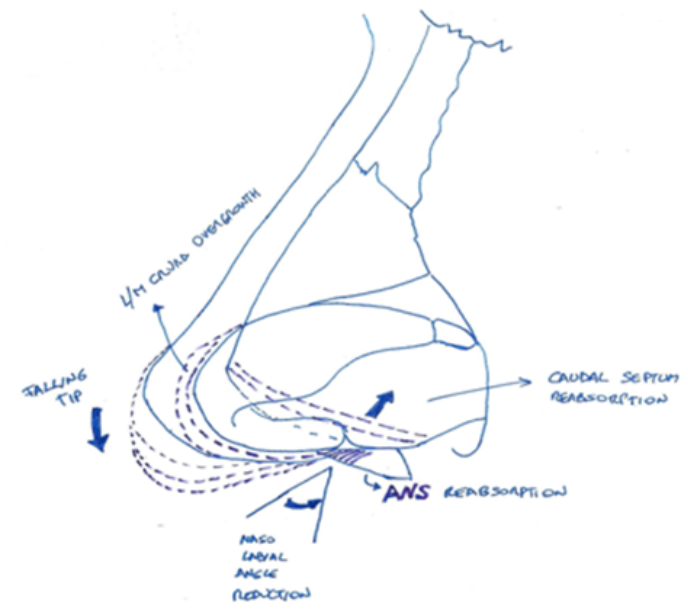

Figure I Physiopathology of Nasal Aging.

\section{Nasal Rejuvenation}

The modern concept of nasal rejuvenation has become lately a matter of interest for physicians in recent international aesthetic meetings, being considered one of the last frontiers of Facial rejuvenation. 
In the past, aesthetic corrections have focus in rejuvenating and improving the external part of the face as done with facelifts. Nevertheless the principal facial traits are located in the center of the face. The former has shifted the aesthetic procedures to a centripetal approach, giving more importance to the eye frame area and nasal procedures.

\section{Nasal rejuvenation procedures}

Prior to 2008, the only available treatments in the nose were surgical. In that setting the etary group seeking for nasal corrections were mostly young individuals. Older patients were not prone to big surgical approaches that would give them a totally different appearance. Moreover their tolerance to swelling, inflammation and downtime decreases as they get into the productive phase. After Braccini's publication, with the introduction of nasal fillers and botulinum toxin, a whole new era of soft nasal beautification techniques, became available.

Very controversial on their introduction they gain more and more interest among the patients seeking for nasal improvements due to lesser invasivity and down time. This treatments were mainly perform over young or middle age individuals, and the techniques available were rudimentary and with high risk of potential devastating complications.

With the apparition of Nasal Torres No-Touch technique on 2015, and the introduction of curved cannula, the repertoire available for nasal corrections widened and the incidence of adverse events dropped dramatically. The former allowed the extension of these corrections to older patients that were not just looking for beautification but to expand their use into nasal aging. The former enlarged the universe of patient seeking for nasal corrections and allowed to propose nasal corrections to patients that otherwise would not have gotten one.

The author has a series of 150 patients above 45 years were all this concepts are applied. The principal techniques used in nasal antiaging include cosmetics, rhino fillers, botulinum toxin, fat grafting, lasers, threads and sutures.

\section{Nasal cosmetics}

Topical preparations applied over the nose can improve hydration, sebometry, reduce fine wrinkles and lines, even the pigmentation and reduce dilated ostium follicular is. The principal active ingredients used include retinoids and chemical peels agents. Their use requires constant exposure and time as results are seen only after 3 months.

\section{Rhinofillers}

The term 'medical rhinoplasty' or 'rhinofiller' is defined as the application of dermal fillers in the external or internal nasal area to modify or improve aesthetics or functionality.

It is especially suitable for patients with minor aesthetic or functional concerns that are refractory to surgery. It may be combined with the use of botulinum toxin injections around the nose to enhance the results. The procedure is currently a frequent request in aesthetic practice, and many physicians perform it systematically. Nevertheless, it should be considered that it is an advanced technique and should only be attempted by expert practitioners due to the potential for devastating vascular complications. Local anatomical knowledge and advanced technical skills are required to achieve successful and safe corrections.

Rhinofiller is an infiltration of a dermal filler to modify external or internal nasal structures for aesthetic or functional purposes. Since its introduction in 2008, many different temporary or permanent substances have been used to achieve the desired corrections. Successful application mandates an adequate anatomical knowledge of the related structures. Proper patient selection is important to achieve good results. Exclusion criteria include: Severe nasal airway impairment, permanent filler in the area, history of ischemic/thrombotic events or known hypercoagulability, local infection, or recent trauma. The indications of rhinofiller (Table 1) are many, including the treatment of nasal aging.

Table I Rhinofiller indications.

\begin{tabular}{|l|}
\hline Rhinofiller Indications \\
\hline Surgery Refractory Pt \\
\hline Absence of Airway Disfunction \\
\hline Small Dorsal Humps \\
\hline Mild Deficit tip Projection \\
\hline Aesthetic Dorsal Lines Asymmetries \\
\hline Small Defect post Rhinoplasty \\
\hline Nasal Aging \\
\hline
\end{tabular}

The fillers around the nose can be a powerful weapon to reestablish volume loss, specially in the radix or base. Moreover they can favor an increase in the nasolabial angle lifting the nose tip and reversing the tip ptosis. The former is achieved with the tripod volumetric graft previously published by the author with the aid of curved cannulas.

Before the procedure, nasal analysis should be performed clinically and photographically, to define needed corrections. Areas of potential correction include aesthetic dorsal lines, dorsum, minor hump camouflage, radix enhancement, tip rotation and projection, and base augmentation. The detail is shown on Figure [2].

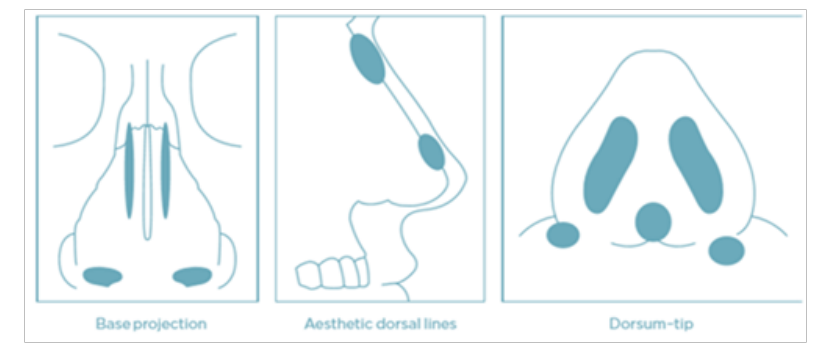

Figure 2 Areas of potential fillers corrections.

Functionally in selected cases, the use of fillers can be useful to augment the aperture of the internal nasal valve, as a volumetric spreader graft.

Morphing simulations are advisable before treatment in order to give patients an indication of the post-treatment outcomes, explain the procedure, and establish common goals. In addition, specific, informed consent should be properly discussed and obtained.

\section{Technique}

Treatments are typically performed with medium viscosity hyaluronic acid fillers, under local anesthetic (lidocaine intradermal vesicles applied using a $0.3 \mathrm{ml}$ syringe with a $32 \mathrm{G}$ needle) with the aid of a $25 \mathrm{G}(0.5 \mathrm{~mm}) \times 4 \mathrm{~cm}$ blunt-tip, disposable cannula, manually bent, maintaining sterility at all times, in order to obtain a better compliance of shapes and silhouette within the nasal area. The distribution of material should be performed as required to follow the treatment plan. Tip refinements can be sporadically carried out through needle infiltration with extreme care. 
The specific pattern of anesthetic peripheral blocks and filler infiltration is shown in Figure 3.
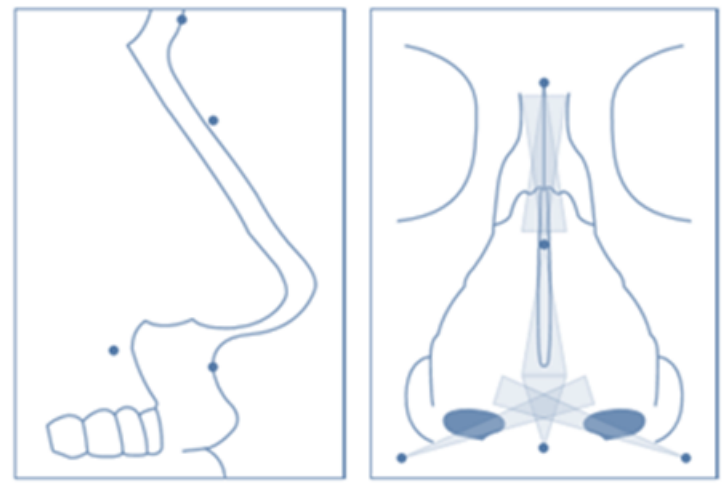

Figure 3 Perispheral Anesthetic blocks and filler distribution.

Generally, patient satisfaction rate with this correction is very high and due to the scarse muscular activity in the nose corrections with Hyaluronic acid dermal fillers last above 1 year and in many cases even two. Rhinofiller clinical cases are shown on Figures 4-6.

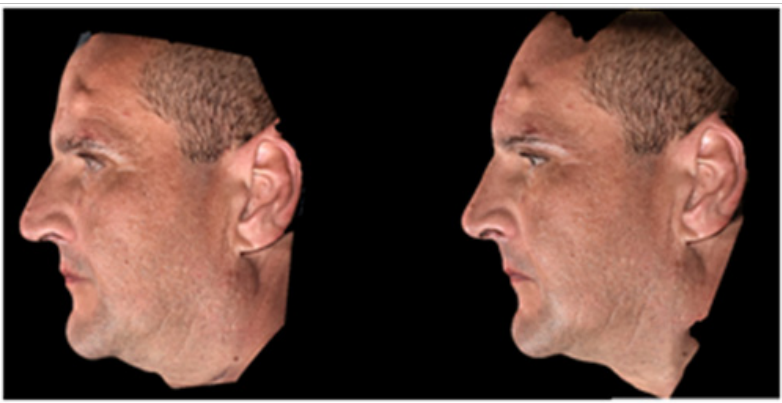

Figure 4 Lateral View of Nasal Rejuvenation with Fillers. Notice straight dorsum, and change in nasolabial angle.

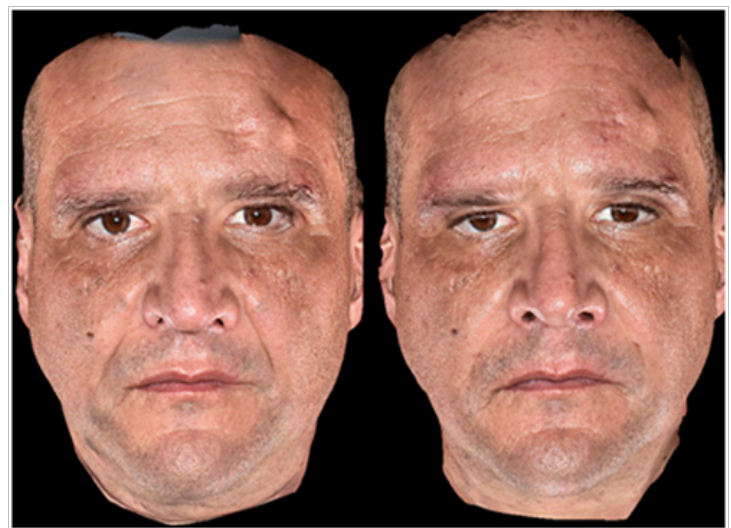

Figure 5 Frontal view of nasal rejuvenation with fillers. Notice the changes in nostrils area exposure.

All of the above have determined nasal augmentation with dermal fillers to be particularly challenging, and mastery of the correct technique is of the utmost importance in order to achieve good results and reduce the incidence of adverse reactions.

\section{Nasal botulinum toxin}

The onset of the neurotoxin in aesthetics revolutionized the treatment of dynamic facial dynamic wrinkles, producing a reversible paralysis that allows overlying tissues to relax and aesthetically to be flattened and raised. The use of botulinum toxin around the nose differs from the typically recommended indications of the superior facial third, being considered an advanced, and off-label technique. The use of botulinum toxin in the nose is useful in hyper-motile noses that typically move with mimic expression. The complications related to this technique are not as severe as with the use of rhinofiller as they are reversible and do not affect nose vascularity. Complications include pain, bruising, swelling, asymmetries, short lasting effect and resistance. Lasting of the corrections is limited (3-4 months) and action takes 2-10 days to establish; but it may enhance the results obtained with a rhinofiller, as it removes muscular action and tension over the nasal region. Deep punctures at a muscular level are necessary. The muscles suitable of being treated around the nose are:

a. Nasalis Transverse muscle is responsible for the wrinkling in the radix paranasal region known as "bunny lines". Treatment typically requires 1-2 Units per side $1 \mathrm{~mm}$ above the angular vessels at the lateral aspect of the radix.

b. Levator Angulis Oris Aleque Nasi muscle is responsible for the gummy smile. Treatment requires $2-5 \mathrm{U}$ per side at the intersection of the nasolabial fold and the alar region.

c. Depressor Septi Nasi muscle is responsible for hypermotile nose tips and acute columellar labial angle. Treatment requires 1-2U at the base of the columella.

d. Alar Nasalis muscle acts together with the Depressor Septi Nasi muscle to lower the tip projection and restrict the nasal aperture. Treatment requires 1-2U per side at the midpoint of the alar area.

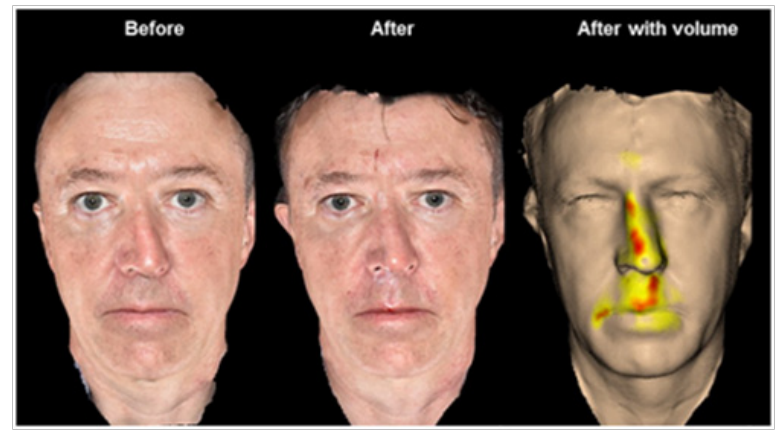

Figure 6 Frontal view of nasal Rejuvenation with fillers. Notice the important nasal deviation corrected after the volumization and the colorimetry design that shows the areas of filler placement.

\section{Nasal lasers}

$\mathrm{CO}_{2}$ Lasers can be extremely useful for the treatment of severy skin hypertrophy such as in rhinophyma. Local anesthetics and sedation are necessary and adequate postop skin care and herpes prophylaxis must be given as healing time can be prolonged.

Vascular lasers such as Ndyag, Dye, KTP or Copper, can be used to treat vascular overgrowth. Tollerance to the procedure is generally good and topical anesthetics are sufficient in most cases. More than one session may be required.

\section{Nasal fat grafting}

Nose lipofilling can be a valid alternative for nasal reconstruction as they can expand the tissues volumetrically with a permanent effect and benefit the tissues due to the regenerative actions of the stem cells within the fat. This can allow further more invasive reconstructive 
techniques, as the tissues recover from previous damages. Micro or nano grafts can be deliver with a technique similar to rhinofillers.

\section{Threads and sutures}

Threads are surgical filaments of generally reabsorbable (PDO, poly lactic acid) materials with self anchoring characteristics that may lift the tissues and stimulate collagen overgrowth. As the threads are free their traction action relies mainly in the tines they have along. They have been used to stimulate volumization through collagen overgrowth, straighten the dorsum and to promote lifting of the tip. Unfortunately results are usually short lasting (3-5 months) as the most ptosic noses are generally bigger, with thicker skin and heavier.

On the other hand nasal sutures are made out of reabsorbable semielastic, braided material (polycaproamide) with deep periosteal anchoring point, the presence of a tie and a knot, that grant greater duration and effect than the threads. The treatments require local anesthetics and sedation and the knots should be performing under medium tension to prevent exaggerated and unnatural effects.

An algorithm to nasal aging and rejuvenation is presented in Figure 7.

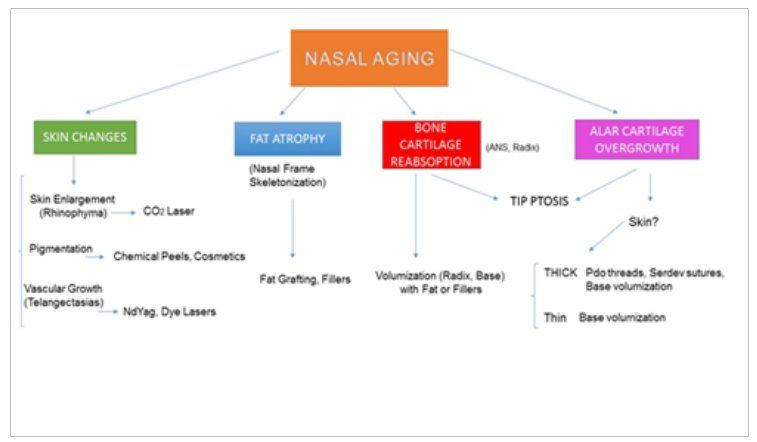

Figure 7 Algorithm of Nasal Aging and Rejuvenation.

\section{Acknowledgements}

Quantificare SA (Valbonne, France) 3D imaging systems, for pictures and visual software support. Adoderm GmbH (Langenfeld, Germany) for Hyaluronic acid fillers (Hyabell) support.

\section{Conflicts of Interest}

None. The author is professional advisor for Adoderm, Germany but receives no economical gain from this publication.

\section{References}

1. Torres S (2015) Nasal volumetric remodeling with the aid of a new stabilized hyaluronic acid dermal filler. EMJ Dermatol 3(1): 98-103.

2. Beiraghi-Toosi A, Rezaei E, Jabbari Nooghabi M, Izadpanah S (2013) Effect of depressor septi nasi muscle activity on nasal lengthening with time. Aesthetic Plast Surg 37(5): 984-988.

3. Mendelson B, Wong CH (2012) Changes in the facial skeleton with aging: implications and clinical applications in facial rejuvenation. Aesthetic Plast Surg 36(4): 753-760.

4. Toriumi DM, Rosenberger E (2016) Rhinoplasty of the Aging Nose Facial Plast Surg 32(1): 59-69.

5. Truswell WH $4^{\text {th }}$ (2013) Aging changes of the periorbita, cheeks, and midface. Facial Plast Surg 29(1): 3-12.
6. Kim SW, Mo JH, Kim JW, Kim DY, Rhee CS, et al. (2007) Change of nasal function with aging in Korean. Acta Otolaryngol Suppl (558): 90-94

7. Guo H, Hu G, Xu Q, Qin H, Li F, et al. (2016) Anthropometric analysis of facial aging in 1200 Han adult people in Dalian. Zhonghua Zheng Xing Wai Ke Za Zhi 32(1): 29-33.

8. Salzano FA, Guastini L, Mora R, Dellepiane M, Salzano G, et al. (2010) Nasal tactile sensitivity in elderly. Acta Otolaryngol 130(12): 13891393.

9. Tüzün Y, Wolf R, Kutlubay Z, Karakuş O, Engin B (2014) Rosacea and rhinophyma. Clin Dermatol 32(1): 35-46.

10. Gassia V, Raspaldo H, Niforos FR, Michaud T (2013) Global 3-dimensional approach to natural rejuvenation: recommendations for perioral, nose, and ear rejuvenation. J Cosmet Dermatol 12(2): 123-136.

11. Humphrey S, Beleznay K, Fitzgerald R (2016) Combination Therapy in Midfacial Rejuvenation. Dermatol Surg 42(Suppl 2): S83-S88.

12. Menick FJ (2013) Practical details of nasal reconstruction. Plast Reconstr Surg 131(4): 613e-630e.

13. Redaelli A, Limardo P (2012) Minimally invasive procedures for nasal aesthetics. J Cutan Aesthet Surg 5(2): 115-120.

14. Karimi K, Adelson RT (2011) The efficacy of rhinoplasty alone in facial rejuvenation. Am J Otolaryngol 32(4): 269-274.

15. Kim SN, Byun DS, Park JH, Han SW, Baik JS, et al. (2014) Panophthalmoplegia and vision loss after cosmetic nasal dorsum injection. J Clin Neurosci 21(4): 678-680.

16. Matros E, Momoh A, Yaremchuk MJ (2009) The aging midfacial skeleton: implications for rejuvenation and reconstruction using implants. Facial Plast Surg 25(4): 252-259.

17. Ries WR, Rathfoot CJ (1996) The aging nose in rhinoplasty for facial rejuvenation. Facial Plast Surg 12(2): 197-203.

18. Hong HR, Kim SH, Kim JH, Jang YJ (2015) Aesthetic Motivation of Geriatric Rhinoplasty: The Surgical Outcome. J Craniofac Surg 26(6): 1936-1939.

19. Kurkjian TJ, Ahmad J, Rohrich RJ (2014) Soft-tissue fillers in rhinoplasty. Plast Reconstr Surg 133(2): 121e-126e.

20. Ozturk CN, Li Y, Tung R, Parker L, Piliang MP, et al. (2013) Complications following injection of soft-tissue fillers. Aesthet Surg J 33(6): 862-877.

21. Moon HJ Use of Fillers in Rhinoplasty. Clin Plast Surg 43(1): 307-317.

22. Sun ZS, Zhu GZ, Wang HB, , Xu X, Cai B, et al. (2015) Clinical Outcomes of Impending Nasal Skin Necrosis Related to Nose and Nasolabial Fold Augmentation with Hyaluronic Acid Fillers. Plast Reconstr Surg 136(4): 434e-441e.

23. Park SW, Woo SJ, Park KH, Huh JW, Jung C, et al. (2012) Iatrogenic retinal artery occlusion caused by cosmetic facial filler injections. Am J Ophthalmol 154(4): 653-662.

24. Youn SH, Seo KK (2016) Filler Rhinoplasty Evaluated by Anthropometric Analysis. Dermatol Surg 42(9): 1071-1081.

25. Kim YJ, Choi KS (2013) Bilateral blindness after filler injection. Plast Reconstr Surg 131(2): 298e-299e.

26. Braccini F, Dohan Ehrenfest DM (2008) Medical rhinoplasty: rationale for atraumatic nasal modelling using botulinum toxin and fillers. Rev Laryngol Otol Rhinol (Bord) 129(4-5): 233-238. 